\title{
Synthesis and Characterization of Doped ZnO Photocatalysts
}

\author{
A.C. Dodd ${ }^{*, *}$, A.J. McKinley ${ }^{*}$, M. Saunders ${ }^{* *}$, and T. Tsuzuki***
}

${ }^{*}$ School of Biomedical and Chemical Sciences, University of Western Australia, 35 Stirling Highway, Crawley, Western Australia 6009

${ }^{* *}$ Centre for Microscopy and Microanalysis, University of Western Australia, 35 Stirling Highway, Crawley, Western Australia 6009

*** Advanced Nanotechnology Limited, 108 Radium Street, Welshpool WA 6106, Australia

Zinc oxide $(\mathrm{ZnO})$ is a semiconductor material with a direct band-gap energy of approximately $3.2 \mathrm{eV}$. As a result, irradiation of $\mathrm{ZnO}$ with sufficiently energetic ultraviolet (UV) light results in the promotion of electrons to the conduction band and the consequent formation of holes in the valence band. These photogenerated charge carriers can subsequently migrate to the surface of the $\mathrm{ZnO}$ and initiate redox reactions with absorbed molecules in a process known as heterogeneous photocatalysis [1].

The phenomenon of heterogeneous photocatalysis has attracted considerable attention as a potentially viable technique for the destruction of intractable chemical waste. However, practical application of photocatalysis for this purpose has been limited by its low quantum yield, which is determined by the rate of interfacial charge transfer relative to that of charge carrier recombination. Increasing the quantum yield of heterogeneous photocatalysis requires a method for inhibiting charge carrier recombination. Previous experimental studies with $\mathrm{TiO}_{2}$ have shown that this can be achieved by the addition of metal ion dopants [2].

We have undertaken a systematic study of the effect of various dopants on the photocatalytic activity of nanoparticulate $\mathrm{ZnO}$. Powders of doped $\mathrm{ZnO}$ were manufactured by a three-stage process consisting of mechanical milling, heat-treatment, and washing. Milling of anhydrous chloride precursors with $\mathrm{Na}_{2} \mathrm{CO}_{3}$ was used to synthesize a composite powder consisting of nanocrystalline carbonate grains dispersed within a matrix of $\mathrm{NaCl}$. Heat-treatment was then used to decompose the carbonate precursor particles to oxide. Finally, the $\mathrm{NaCl}$ by-product was removed by washing with deionised water. This sequence is illustrated by x-ray diffraction (XRD) patterns of a $\mathrm{ZnCl}_{2}+\mathrm{Na}_{2} \mathrm{CO}_{3}+4 \mathrm{NaCl}$ reactant mixture following each processing stage, as shown in Figure 1.

The mechanochemical synthesis technique allows significant control to be exercised over the characteristics of the final washed powder. For example, the average particle size can readily be controlled through the temperature of the post-milling heat treatment. Furthermore, the simultaneous formation of the nanoparticles with an intervening salt matrix inhibits agglomerate formation since the salt matrix inherently separates the particle from each other during processing. This illustrated in Figure 2, which shows a representative bright field TEM image of a washed $\mathrm{ZnO}$ powder that was synthesized by mechanochemical processing. 
While bright field imaging allows us to study the particle size and agglomeration, it does not provide direct information on the presence and role of the dopants in the material. Therefore, EFTEM and EELS have been used to characterize the dopant distribution at the nanoscale, with the objective of determining whether the dopants are incorporated into the $\mathrm{ZnO}$ as a solid solution or if they form a separate phase. In addition, variations in the role of the dopants as a function of the applied heat treatment have also been investigated.

The photocatalytic activity of the doped $\mathrm{ZnO}$ powders was characterized by measuring the hydroxyl radical concentration as a function of irradiation time using the spin trapping technique with electron paramagnetic resonance (EPR) spectroscopy [3]. The ultimate goal of this work is to correlate the microscopy data with the EPR results as part of an ongoing effort to develop methods for controlling the photocatalytic activity of nanoparticulate $\mathrm{ZnO}$.

\section{References}

[1] M. Hoffmann et al., Chem. Revs., Vol. 95 (1995), p 69.

[2] Z. Zhang et al., J. Phys. Chem. B, Vol. 102 (1998), p 10871.

[3] M. Grela et al., J. Phys. Chem., Vol. 100 (1996), p 16940.

[4] This research was supported by an Australian Research Council Linkage grant LP0349177
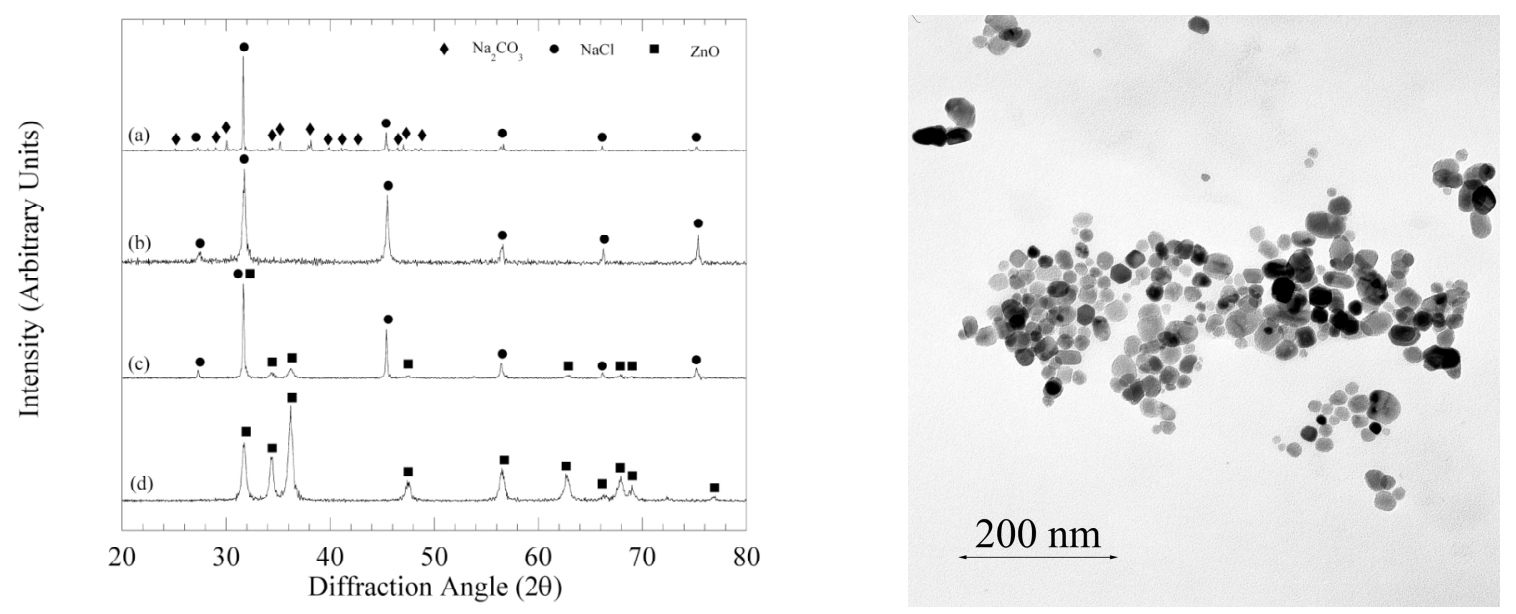

Fig. 1: XRD pattern of $\mathrm{ZnCl}_{2}+\mathrm{Na}_{2} \mathrm{CO}_{3}+4 \mathrm{NaCl}$ following (a) mixing, (b) milling, (c) heat-treatment, and (d) washing.

Fig. 2: Bright field TEM image of a washed $\mathrm{ZnO}$ powder that was synthesized by mechanochemical processing. 